\title{
Haemorrhagic Transformation after Ischaemic Stroke in Patients Taking Non-vitamin K Antagonist Oral Anticoagulants
}

\author{
Jan C. Purrucker, ${ }^{\mathrm{a}}$ Kirsten Haas, ${ }^{\mathrm{b}}$ Marcel Wolf, ${ }^{\mathrm{c}}$ Timolaos Rizos, ${ }^{\mathrm{a}}$ Shujah Khan, ${ }^{\mathrm{a}}$ Peter Kraft, ${ }^{\mathrm{d}}$ Sven Poli, \\ Rainer Dziewas, ${ }^{\mathrm{f}}$ Johannes Meyne, ${ }^{\mathrm{g}}$ Frederick Palm, ${ }^{\mathrm{h}}$ Sebastian Jander, ${ }^{\mathrm{i}}$ Markus Möhlenbruch, ${ }^{\mathrm{c}}$ \\ Peter U. Heuschmann, ${ }^{\mathrm{b}, \mathrm{j}}$ and Roland Veltkamp, ${ }^{\mathrm{a}, \mathrm{k}}$ on behalf of the RASUNOA investigators \\ ${ }^{a}$ Department of Neurology, Heidelberg University Hospital, Heidelberg, Germany \\ 'Institute of Clinical Epidemiology and Biometry, University Würzburg, Germany \\ 'Department of Neuroradiology, Heidelberg University Hospital, Heidelberg, Germany \\ dDepartment of Neurology, University Hospital Würzburg, Würzburg, Germany \\ 'Department of Neurology, Tübingen University, Tübingen, Germany \\ fDepartment of Neurology, University Hospital Münster, Germany \\ ${ }^{9}$ Department of Neurology, University Hospital Schleswig-Holstein, Kiel, Germany \\ hDepartment of Neurology, Klinikum Ludwigshafen, Ludwigshafen, Germany \\ 'Department of Neurology, Heinrich-Heine-University, Medical Faculty, Düsseldorf, Germany \\ 'Comprehensive Heart Failure Center, and Clinical Trial Center, University Hospital Würzburg, Würzburg, Germany \\ kDepartment of Stroke Medicine, Imperial College London, London, United Kingdom
}

Background and Purpose To evaluate the frequency and outcome of haemorrhagic transformation (HT) after ischaemic stroke in patients treated with non-vitamin $\mathrm{K}$ antagonist oral anticoagulants (NOACs).

Methods Patients with stroke on treatment with a NOAC were prospectively enrolled in this multicentre observational study between February 2012 and 2015. Brain imaging at admission and followup imaging until day 7 were reviewed for HT. Functional outcome was assessed by the modified Rankin scale (mRS) before the index event, at discharge, and at 3-months.

Results 231 patients without recanalisation therapy (no-RT), and 32 patients with RT were eligible for analysis. Any HT was present at admission in 9/231 no-RT patients (3.9\%, 95\% Cl 2.0 to 7.3 ) and in none of the patients with RT. In patients with follow-up imaging (no-RT, $n=129$, and $R T, n=32$ ), HT was present in 14.0\% (no- $\mathrm{RT}$; $95 \% \mathrm{Cl}, 8.9$ to 21.1 ), and $40.6 \%$ (RT, 95\% Cl, 25.5 to 57.8 ), respectively. After adjustment for stroke severity, this difference between the no-RT and RT groups became nonsignificant. Symptomatic ICH was observed in 1 patient per group. HT was not associated with unfavourable outcome (mRS 3-6) at 3-months in multivariable analysis. Resumption of OAC after stroke was delayed in patients with $\mathrm{HT}$ compared to those without (15 d [IQR, 5-26] vs. $1 \mathrm{~d}[0-4], P<0.001$ ). Conclusions The frequency and severity of $\mathrm{HT}$ after stroke on NOAC appears similar to previous reports for vitamin $\mathrm{K}$ antagonists and no anticoagulation. Whether asymptomatic HT should delay resumption of preventive anticoagulation requires further investigation.
Correspondence: Roland Veltkamp Department of Stroke Medicine, Imperial College London, Charing Cross Campus, 3 East 6, Fulham Palace Road, London, W6 8RF, United Kingdom Phone: +44-20-33130133, Fax: +44-20-83833309, E-mail: r.veltkamp@imperial.ac.uk

Received: June 16, 2016 Revised: October 11, 2016 Accepted: November 28, 2016

The authors have no financial conflicts of interest.

Keywords Stroke, Anticoagulation, Haemorrhagic Transformation 


\section{Introduction}

Any haemorrhagic transformation (HT) is found in approximately $8.5 \%$ (95\% $\mathrm{Cl} 7-10 \%)$, of non-thrombolysed acute ischaemic stroke (AIS) patients. ' Symptomatic HT associated with clinical worsening or death occurs in 1.5\% (95\% $\mathrm{Cl} 0.8-2.2 \%)^{1.2}$ In the randomised European Cooperative Acute Stroke Study III, the rate of any HT was notably higher in both the IV thrombolysis and placebo-arm (27.0\% vs. $17.6 \%)$. ${ }^{3}$ However, differences in assessment criteria and $\mathrm{HT}$ definitions hamper direct comparisons. ${ }^{4}$ While oral anticoagulation (OAC) with vitamin $\mathrm{K}$ antagonists (VKA) does not appear to affect the occurrence of $\mathrm{HT}_{1}^{2,5}$ data regarding the frequency and severity of $H T$ in patients treated with non-Vitamin $\mathrm{K}$ antagonist oral anticoagulants (NOACS) is not available. The potential effects of HT on neurological outcome and on the interval until re-initiation of anticoagulation are unknown.

We evaluated the frequency and severity of $\mathrm{HT}$ at admission and during the first 7 days in acute stroke patients taking NO$\mathrm{ACs}$, and examined effects on the management and functional outcome of AIS under NOAC treatment using data from the multicentre Registry of Acute Stroke Under New Oral Anticoagulants (RASUNOA-pilot).

\section{Methods}

\section{Study design, setting and variables}

Patients presenting between February 2012 and February 2015 with an acute ischaemic stroke and taking NOACs at the time of the event were prospectively enrolled into the RASUNOA-pilot registry, an investigator-initiated, multicentre, observational cohort study (ClinicalTrials.gov, NCT01850797). Overall, 38 neurology departments with certified stroke units across Germany participated in the registry. Inclusion criteria were age $\geq 18$ years, acute ischaemic stroke, and current therapy with a NOAC (i.e., Apixaban, Dabigatran or Rivaroxaban) at the time of stroke onset. For the present analysis, we excluded (i) patients without any available brain imaging, and (ii) patients with transient ischaemic attack (as defined by presentation without neurological deficit according to the National Institute of Health Stroke Scale (NIHSS) at the time of admission and absence of an acute ischaemic lesion on brain imaging ${ }^{6}$ ). Due to the observational character of the registry, all diagnostic and treatment decisions were left to the discretion of the attending physicians. Patient characteristics including demographic information, clinical data and laboratory parameters were prospectively collected using a standardized case report file. The modified Rankin scale score (mRS) was used for functional assessment prior to the stroke (pmRS), at admission, and at hospital discharge. Moreover, 3-month outcome was assessed by a structured telephone follow-up. The individual stroke risk in patients with atrial fibrillation was calculated using the $\mathrm{CHA}_{2} \mathrm{DS}_{2} \mathrm{VASc}$-score (excluding the index event), and the individual bleeding risk using the HAS BLED-score, respectively, with the item "labile INR" set to zero.,

The analysis was done stratified for patients not undergoing thrombolysis and/or thrombectomy (no-RT) and patients who received recanalisation therapy (RT), respectively (a focused analysis of the RT group without IV-only treated patients has been previously published. ${ }^{9}$

\section{Neuroimaging analysis}

Cranial CT and MRI examinations were assessed for signs of ischaemic infarction, as well as intracranial haemorrhage by an experienced reader (JP). Assessment was performed on scans obtained at admission, and on follow-up scans carried out until day 7 if available. Regarding diagnosis and classification of haemorrhage, findings were reviewed by a board-certified neuroradiologist (MW). Disagreement between the two readers was resolved by consensus. Readers were blinded for patient details. HT was defined as any level of hyperdensity within the area of low attenuation (CT), or hypointensities on $\mathrm{T}^{*}$ or susceptibility weighted (SWI) sequences ${ }^{10}$ in areas corresponding to DWI/FLAIR hyperintense regions on MRI. If rapid attenuation of initial hyperdensities on CT scans was observed, this was categorized as contrast staining rather than hemorrhage. ${ }^{9}$

Haemorrhagic transformation was classified in accordance with the ECASS-I-criteria as either haemorrhagic infarction (HI1 and 2), or parenchymal haemorrhage (PH1 and 2)." Accordingly, HI1 was defined as small petechiae along the margin of the infarct, HI2 as more confluent petechiae within the infarct but without space-occupying effect, $\mathrm{PH} 1$ as a blood clot not exceeding 30\% of the infarct area with some mild space-occupying effect, and PH2 as blood clots exceeding 30\% of the infarct area with significant space-occupying effect (Figure 1B). ${ }^{11}$ Moreover, we recorded intracerebral haemorrhage remote from the infarct, which was defined as small or medium-sized blood clots (PHr1) or large confluent dense blood clots (PHr2). Evidence of intraventricular or subarachnoidal haemorrhage was also captured. Symptomatic intracranial haemorrhage ( $\mathrm{slCH}$ ) was defined following the ECASS-II definition. ${ }^{12}$ The extent of infarction was quantified using the Alberta Stroke Program Early Computed Tomography Score (ASPECTS; range 0 to 10 with 1 point subtracted for any evidence of ischaemic change in each defined region on axial cuts). In case of posterior circulation stroke, the pc-ASPECTS score was calculated. ${ }^{13,14}$ For quantification of the site and size of ischaemic infarction the classification by Paciaroni and 


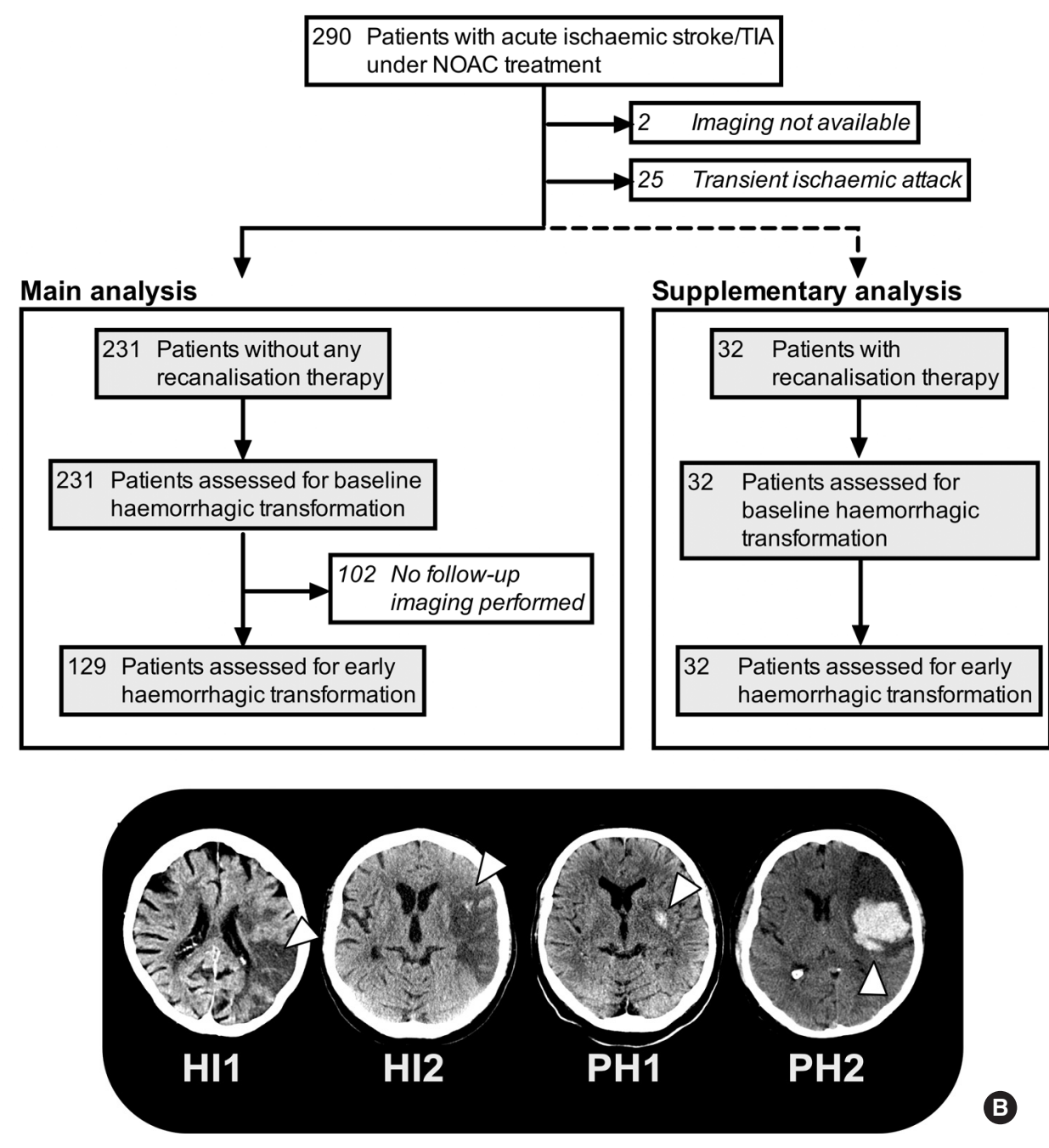

Figure 1. Selection of study population and classification of haemorrhagic transformation. (A) Study flow chart. (B) Representative computed tomography scans showing the four types of haemorrhagic transformation. HI, haemorrhagic infarction; $\mathrm{PH}$, parenchymal haematoma.

colleagues was used. ${ }^{2}$ Accordingly, infarct size was classified as (1) small (lesion in the anterior or posterior circulation $<1.5 \mathrm{~cm}$ ), (2) medium (lesion in a cortical superficial branch of middle cerebral artery [MCA], or lesion involving the MCA deep branch, or lesion in internal borderzone territories, or lesion in a cortical superficial branch of posterior cerebral artery [PCA], or lesion involving the PCA branch or lesion in a cortical superficial branch of anterior cerebral artery [ACA]), (3) large anterior (lesion involving complete territory of MCA, PCA or ACA or lesion involving 2 cortical superficial branches of MCA or lesion involving a cortical superficial branch of MCA associated to the MCA deep branch, or lesion involving more than 1 artery territory [e.g., MCA associated to ACA territories]) or (4) large posterior (lesion involving brain stem or cerebellum $>1.5 \mathrm{~cm}$ ). ${ }^{2}$ Cases without definitive evidence of acute cerebral infarction on available scans were added to the Paciaroni category 1.

\section{Statistical analysis}

All analyses were descriptive and exploratory. Continuous variables were described by mean and standard deviation (SD) or median and interquartile-range (IQR) for categorical variables, absolute and relative frequencies were reported. The ShapiroWilk test was used to ascertain distribution of data. Post-hoc, groups of patients with haemorrhagic transformation (HT) and without (non-HT) were defined. Fishers-exact test was used to compare proportions in baseline and radiological characteristics, dosing categories (standard [apixaban $5 \mathrm{mg} \mathrm{BID}$, dabigatran 150 mg BID, rivaroxaban $20 \mathrm{mg} \mathrm{OD}$ ] vs. low drug dose[apixaban 2.5 mg BID, dabigatran $110 \mathrm{mg} \mathrm{BID}$, rivaroxaban $15 \mathrm{mg}$ QD], and considering the actual dose, current renal function and age, adequate or overdosing) and outcomes, as appropriate. To compare continuous variables, t-test or non-parametric Mann-Whitney $\mathrm{U}$ test was used according to the skewness of the data. To calculate 95\% confidence intervals (CI) of proportions, the adjusted Wald 
method was used..$^{15}$ Logistic regression analysis were performed to estimate odds ratio (OR) and $95 \% \mathrm{Cl}$ for potential risk factors of HT occurrence or unfavourable 3-months-outcome (mRS 3-6). In multivariable analysis the independent effect of HT on unfavourable 3-months-outcome adjusted for a subset of risk factors was assessed. Demographic variables and variables significantly related to outcome in the univariate analyses were included in the model under avoidance of possible collinearity. For variable selection the stepwise backward method with likelihood ratio test was used. Main characteristics of patients from the five best recruiting centres were compared to the standard recruiting ones in a sensitivity analysis. All statistical tests were two-sided, and $P$ values of $<0.05$ were considered statistically significant. Analyses were conducted using IBM SPSS Statistics, version 23 (IBM Corp., Armonk, NY, USA). This study was performed consistent with the STROBE guidelines for observational studies.

\section{Standard protocol approvals, registrations, and patient consents}

The study was approved by the ethics committee of the Medical Faculty Heidelberg, Germany, and the ethics committees of each participating centre. Written informed consent by either the patient or a legal representative was mandatory for participation. The institutional review board at the primary study site in Heidelberg approved a waiver of consent for patients who died during initial hospitalization and for whom a legal representative could not be located.

\section{Results}

\section{Study population and baseline characteristics}

Of the 290 patients with acute ischaemic stroke/TIA under NOAC treatment enrolled in RASUNOA-pilot, 231 patients were included in the haemorrhagic transformation main analysis group (patient flow in Figure $1 \mathrm{~A}$ ) and 32 patients who were treated with recanalisation therapies ( $R T ; n=23$ endovascular therapy alone, $n=5$ IV thrombolysis plus thrombectomy, $n=4$ IV thrombolysis alone) were analysed separately (Figure $1 \mathrm{~A}$ ).

Baseline characteristics of the no-RT group are presented in Table 1, and of the RT group in the online Supplementary Table 1. In the no-RT group, the mean age of included patients was 77 (SD 8.4) years, and $46.8 \%$ were female. Median onset to first brain imaging was 3.4 hours (IQR, 2.1-9.9; exact onset of symptoms was available in $n=156$ ). Most patients were affected by minor or moderate stroke (median NIHSS 3 [IOR 2-6]). Nineteenpercent suffered from large anterior or posterior strokes (Table 2).

Any follow-up imaging until day 7 was performed in 55.8\% (129/231) of the no-RT group. The median time between scans was 49.6 hours (IQR, 22.4-86.0). Baseline characteristics of patients with follow-up imaging did not differ from those without (Supplementary Table 2).

Compared to patients with follow-up imaging and no recanalisation therapy (no-RT), RT-patients were more severely affected (NIHSS at admission 19 [IQR 14-22] vs. 4 [IQR 2-7], $P<0.01$ ) and suffered from larger baseline ischaemic lesions (ASPECTS 8 [IQR 7-10] vs. 10 (9-10, $P<0.01$; Supplementary Table 1).

\section{Haemorrhagic transformation - No-RT group}

At admission, HT was present in 9/231 patients of the no-RT group (initial-HT, 3.9\%, 95\% Cl, 2.0 to 7.3; Table 2), and in none of the RT group. A strong trend towards a higher rate of concomitant antiplatelet therapy was observed in the initial-HT patients (33\% vs. $9.5 \%$; $P=0.06$; Table 1 ). In patients with followup imaging $(n=129), 13$ additional HTs were observed until day 7 (Table 2). In total, 18 cases of HT were registered among patients with available initial and follow-up imaging (early-HT, $18 / 129,14.0 \%, 95 \% \mathrm{Cl}, 8.9$ to 21.1$)$.

There was no association between standard vs. low drug dose, or adequate or overdosing (considering actual dose and current renal function and age) and occurrence of initial-HT or early-HT in patients with AF. Univariate logistic regression revealed that infarct size had the strongest impact on the occurrence of early$\mathrm{HT}$ (size small vs. medium OR 15.2, 95\% $\mathrm{Cl} 2.8$ to 82.7 ; small vs. large OR 19.0, 95\% Cl 3.9 to 93.7, Table 3).

Symptomatic HT occurred in one patient in the follow-up-imaging study population $\left(1 / 129,0.8 \%, 95 \% \mathrm{Cl}_{1}<0.1\right.$ to $4.7 \%$; Table 2). This patient suffered from a large MCA-territory infarction but did not show signs of HT on the initial CT-scan. The NOAC was continued after the stroke and a $\mathrm{PH} 2$ bleeding developed on day 2. All other observed HT remained asymptomatic.

\section{Haemorrhagic transformation - RT group}

In an unadjusted analysis, HT was more frequent in patients treated with thrombolysis and/or thrombectomy compared to those without ( $n=13 / 32,40.6 \%[95 \% \mathrm{Cl}, 25.5$ to 57.8$)$ vs. $18 / 129,14.0 \%$ [95\% $\mathrm{Cl}, 8.9$ to 21.1$], P=0.001)$. No HT was observed in the patients treated with IV thrombolysis only $(n=4)$. However, differences in HT-rates are likely due to higher stroke severity (Supplementary Table 1) in patients treated with recanalisation therapy (after adjustment for stroke severity according to the admission NIHSS, logistic regression for the occurrence on HT became non-significant).

\section{Association with functional outcome - No-RT group}

Patients with early-HT were more severely affected at admission (median NIHSS 7 [IOR, 2-19] vs. 3 [IOR, 2-6], P= 0.03; Table 
Table 1. Baseline characteristics and functional outcome variables of patients with and without haemorrhagic transformation (no-RT group)

\begin{tabular}{|c|c|c|c|c|c|c|c|}
\hline & \multirow[b]{2}{*}{ All Patients } & \multicolumn{3}{|c|}{ Admission imaging cohort } & \multicolumn{3}{|c|}{ Follow-up imaging cohort } \\
\hline & & $\begin{array}{l}\text { Without } \\
\text { initial-HT }\end{array}$ & Initial-HT & $P$ value & $\begin{array}{l}\text { Without } \\
\text { early-HT }\end{array}$ & Early-HT & $P$ value \\
\hline $\mathrm{N}$ & 231 & 222 & 9 & & 111 & 18 & \\
\hline Age (year) & $77.4( \pm 8.4)$ & $77.5( \pm 8.4)$ & $75.4( \pm 8.3)$ & 0.45 & $77.2( \pm 8.2)$ & $76.1( \pm 5.6)$ & 0.47 \\
\hline Women & $108(46.8)$ & $106(47.7)$ & $2(22.2)$ & 0.18 & $57(51.4)$ & 7 (38.9) & 0.45 \\
\hline NOAC & & & & 0.86 & & & 0.29 \\
\hline Apixaban & $36(15.6)$ & 35 (15.8) & $1(11.1)$ & & $19(17.1)$ & $1(5.6)$ & \\
\hline Dabigatran & $62(26.8)$ & $59(26.6)$ & $3(33.3)$ & & $32(28.8)$ & $4(22.2)$ & \\
\hline Rivaroxaban & $133(57.6)$ & $128(57.7)$ & $5(55.6)$ & & $60(54.1)$ & $13(72.2)$ & \\
\hline Time since last intake NOAC (hour) & $8.7(4.3-15.2)$ & $8.8(4.3-15.4)$ & $5.9(4.4-18.2)$ & 0.87 & $9.0(4.2-18.1)$ & $12.8(4.5-20.8)$ & 0.48 \\
\hline Time until resumption of OAC (day) & $1(0-3)$ & $1(0-3)$ & $2(1-15)$ & 0.017 & $1(0-3)$ & $15(5-26)$ & $<.001$ \\
\hline Concomitant platelet inhibition & $24(10.4)$ & $21(9.5)$ & $3(33.3)$ & 0.06 & $10(9.0)$ & $3(16.7)$ & 0.39 \\
\hline $\mathrm{CHA}_{2} \mathrm{DS}_{2}$ VASc score ${ }^{*}$ & $5(3-6)$ & $5(3-6)$ & $4(3-8)$ & 0.63 & $5(4-6)$ & $4(3-6)$ & 0.24 \\
\hline HAS-BLED ${ }^{+}$ & $4(3-4)$ & $4(3-4)$ & $3(3-4)$ & 0.52 & $4(3-4)$ & $3(3-4)$ & 0.09 \\
\hline \multicolumn{8}{|l|}{ Medical history ${ }^{\mp}$} \\
\hline Ischaemic stroke/TIA & $107(46.3)$ & $102(45.9)$ & $5(55.6)$ & 0.74 & $53(47.7)$ & $7(38.9)$ & 0.61 \\
\hline Intracranial Haemorrhage & $5(2.2)$ & $5(2.3)$ & $0(0)$ & $>0.99$ & $3(2.7)$ & $0(0)$ & $>0.99$ \\
\hline Atrial fibrillation & $209(90.5)$ & $200(90.1)$ & $9(100)$ & $>0.99$ & $101(91.0)$ & $18(100)$ & 0.36 \\
\hline Hypertension & $193(83.5)$ & $186(83.8)$ & $7(77.8)$ & 0.65 & 93 (83.8) & $13(72.2)$ & 0.32 \\
\hline Hyperlipidemia & $81(35.1)$ & $80(36.0)$ & $1(11.1)$ & 0.17 & $42(37.8)$ & $5(27.8)$ & 0.60 \\
\hline Diabetes mellitus & 69 (29.9) & $63(28.4)$ & $6(66.7)$ & 0.02 & $38(34.2)$ & $6(33.3)$ & $>0.99$ \\
\hline Heart failure & $53(22.9)$ & $51(23.0)$ & $2(22.2)$ & $>0.99$ & $28(25.2)$ & $6(33.3)$ & 0.57 \\
\hline Peripheral vascular disease & $15(6.5)$ & $14(6.3)$ & $1(11.1)$ & 0.46 & $8(7.2)$ & $1(5.6)$ & $>0.99$ \\
\hline \multicolumn{8}{|l|}{ Renal function } \\
\hline $\mathrm{GFR}<60 \mathrm{~mL} / \mathrm{min}$ & $85 / 206$ (41.3) & 82/197 (36.9) & $3(33.3)$ & 0.74 & 45/99 (45.5) & $8 / 18(44.4)$ & $>0.99$ \\
\hline Creatinin level (mg/dL) & $1.0(0.9-1.3)$ & $1.0(0.9-1.3)$ & $1.0(0.7-1.2)$ & 0.37 & $1.0(0.8-1.3)$ & $1.0(0.9-1.2)$ & 0.95 \\
\hline NIHSS at admission & $3(2-6)$ & $3(2-6)$ & $4(1-7)$ & 0.87 & $3(2-6)$ & $7(2-19)$ & 0.03 \\
\hline \multicolumn{8}{|l|}{ Modified Rankin scale score } \\
\hline Pre stroke & $1(0-2)$ & $1(0-2)$ & $2(1-3)$ & 0.14 & $1(0-2)$ & $1(1-2)$ & 0.13 \\
\hline At admission & $3(1-4)$ & $3(1-4)$ & $4(1-5)$ & 0.21 & $2(1-4)$ & $5(3-5)$ & 0.001 \\
\hline At discharge & $2(1-3)$ & $2(1-3)$ & $3(2-5)$ & 0.15 & $2(1-3)$ & $4(2-5)$ & 0.001 \\
\hline At 3-months ${ }^{\S}$ & $2(1-4)$ & $2(1-4)$ & $2(1-6)$ & 0.78 & $1(1-4)$ & $4(1-6)$ & 0.001 \\
\hline mRS 0-2 at 3-months & $110(53.1)$ & $105(47.3)$ & $5(55.6)$ & $>0.99$ & $60(60.6)$ & $6(33.3)$ & 0.04 \\
\hline In-hospital mortality & $6 / 229(2.6)$ & $6 / 220(2.7)$ & $0(0)$ & $>0.99$ & $2(1.8)$ & $2(11.1)$ & 0.09 \\
\hline
\end{tabular}

Data are mean $( \pm S D)$, median (IQR) or $n(\%)$. Missing data for creatinin level $(n=5)$, GFR $(n=25), m R S$ at discharge $(n=2)$ and at 3-months $(n=24)$, time until resumption of anticoagulation, $(n=29)$.

NOAC, non-vitamin K antagonist oral anticoagulant; TIA, transient ischaemic attack; GFR, glomerular filtration rate (electronic GFR as reported by the centres); NIHSS, National Institutes of Health Stroke Scale; mRS, modified Rankin score [from 0 (no symptoms) to 6 (death)]; no-RT, no recanalisation therapy group. ${ }^{*} \mathrm{CHA}_{2} \mathrm{DS}_{2}$ VASc score, range 0-9, from low to high risk of ischemic stroke in atrial fibrillation; ${ }^{+} \mathrm{HAS}-\mathrm{BLED}$ score, range $0-9$, from low to high risk of haemorrhage under oral anticoagulation; ${ }^{\dagger}$ Medical history, excluding the index-event; ${ }^{5} 3$-months modified Rankin data available for 207/231 patients (89.6\%).

1), and suffered more frequently from larger anterior and posterior infarctions than patients without early-HT $(P<0.001$; Table 2). Patients with early-HT had a significantly worse outcome compared to those without (median mRS 4 [IQR, 1-6] vs. mRS 2 [IOR 1-4], $P=0.001$; Table 1). However, in multivariate analysis, HT was not independently associated with unfavourable outcome (mRS 3-6) at 3-months (Table 4; for univariate analysis, see Supplementary Table 3). In-hospital mortality was not significantly increased among patients with $\mathrm{HT}$.

\section{Resumption of OAC - No-RT group}

In stroke survivors, OAC was resumed during the acute inpa- tient stay in the majority of patients $(n=184 / 230,80 \%$; thereof 97\% NOAC, 3\% phenprocoumon). In patients with early-HT, $\mathrm{OAC}$ was resumed after occurrence of $\mathrm{HT}$ in all but one case. Median time to recommended resumption was significantly longer in patients with early-HT (15d [IOR, 5-26]) compared to patients without ( $1 \mathrm{~d}[0-4] ; P<0.001$ ). Notably, the delay was at least partly mediated through larger infarct-sizes in the early-HT group (see above), as a generally longer time until resumption of OAC was observed in patients without HT but large infarct compared to patients without $\mathrm{HT}$ and a small infarct (10 d [IQR, $0-15]$ and $1 \mathrm{~d}[I \mathrm{QR}, 0-2]_{;} P=0.034$; data not shown). 
Table 2. Radiological characteristics and outcomes (no-RT group)

\begin{tabular}{|c|c|c|c|c|c|c|}
\hline & \multicolumn{3}{|c|}{ Admission imaging cohort } & \multicolumn{3}{|c|}{ Follow-up imaging cohort } \\
\hline & Without initial-HT & Initial-HT & $P$ value & Without early-HT & Early-HT & $P$ value \\
\hline $\mathrm{N}$ & 222 & 9 & - & 111 & 18 & - \\
\hline MRI available & 118 (53.2) & 3 (33.3) & 0.32 & 76 (68.5) & $9(50.0)$ & 0.18 \\
\hline Proximal vessel occlusion* & 21/121 (17.4) & $0 / 2(0)$ & $>0.99$ & 11/69 (15.9) & $4 / 9(44.4)$ & 0.06 \\
\hline Infarct size & & & 0.001 & & & $<0.001$ \\
\hline Small & $148(66.7)$ & $0(0)$ & - & $76(68.5)$ & $2(11.1)$ & - \\
\hline Medium & $36(16.2)$ & $4(44.4)$ & - & $15(13.5)$ & $6(33.3)$ & - \\
\hline Large anterior & $29(13.1)$ & $4(44.4)$ & - & $16(14.4)$ & $8(44.4)$ & - \\
\hline Large posterior & $9(4.1)$ & $1(11.1)$ & - & $4(3.6)$ & $2(11.1)$ & - \\
\hline \multicolumn{7}{|l|}{ Admission imaging } \\
\hline ASPECTS & $10(9-10)$ & $9(8-9)$ & 0.003 & $10(9-10)$ & $9(5-9)$ & $<0.001$ \\
\hline $\mathrm{HI} 1$ & $0(0)$ & $6(66.7)$ & - & $0(0)$ & $2(11.1)$ & - \\
\hline $\mathrm{HI} 2$ & $0(0)$ & $3(33.3)$ & - & $0(0)$ & $3(16.7)$ & - \\
\hline $\mathrm{PH} 1$ & $0(0)$ & $0(0)$ & - & $0(0)$ & $0(0)$ & - \\
\hline $\mathrm{PH} 2$ & $0(0)$ & $0(0)$ & - & $0(0)$ & $0(0)$ & - \\
\hline $\mathrm{PHr} 1 / \mathrm{PHr} 2$ & $0(0) / 0(0)$ & $0(0) / 0(0)$ & - & $0(0) / 0(0)$ & $0(0) / 0(0)$ & - \\
\hline $\mathrm{SAH}$ & $0(0)$ & $0(0)$ & - & $0(0)$ & $0(0)$ & - \\
\hline IVH & $0(0)$ & $0(0)$ & - & $0(0)$ & $0(0)$ & - \\
\hline $\mathrm{sICH}$ & $0(0)$ & $0(0)$ & - & $0(0)$ & $0(0)$ & - \\
\hline \multicolumn{7}{|l|}{ Follow-up imaging until day 7} \\
\hline ASPECTS & - & - & - & $9(8-10)$ & $5(4-9)$ & 0.001 \\
\hline $\mathrm{HI} 1$ & - & - & - & $0(0) / 0(0)$ & $10(55.6)$ & - \\
\hline $\mathrm{HI} 2$ & - & - & - & $0(0)$ & $2(11.1)$ & - \\
\hline $\mathrm{PH} 1$ & - & - & - & $0(0)$ & $0(0)$ & - \\
\hline $\mathrm{PH} 2$ & - & - & - & $0(0)$ & $1(5.6)$ & - \\
\hline $\mathrm{PHr} 1 / \mathrm{PHr} 2$ & - & - & - & $0(0)$ & $0(0) / 0(0)$ & - \\
\hline $\mathrm{SAH}$ & - & - & - & $0(0)$ & $0(0)$ & - \\
\hline IVH & - & - & - & $0(0)$ & $0(0)$ & - \\
\hline $\mathrm{sICH}$ & - & - & - & $0(0)$ & $1(5.6)$ & - \\
\hline
\end{tabular}

Data are median (IQR) or $n(\%)$. Note: In the follow-up imaging group, a total of 5 haemorrhages were present at admission, and 13 additional haemorrhages became present during follow-up imaging, resulting in a total of 18 hemorrhagic transformations in this group.

MRI, magnetic resonance imaging; ASPECTS, Alberta Stroke Program Early Computed Tomography Score; HI1/2, haemorrhagic infarction type 1/2; PH1/2, parenchymal haemorrhage type 1/2; SAH, subarachnoid haemorrhage; IVH, intraventricular haemorrhage; sICH, symptomatic intracerebral haemorrhage (according to the ECASS-II criteria); no-RT, no recanalisation therapy group.

${ }^{*} \mathrm{CT}$, MR or digital subtraction angiography available in 123/231 patients (53.2\%).

\section{Discussion}

The new findings of our study are that (1) spontaneous HT occurs at a similar rate in acute stroke patients treated with NOAC as previously reported for non-anticoagulated and VKA-anticoagulated stroke patients, ${ }^{1,2,5}(2)$ recanalisation therapy did not increase the proportion of patients with HT after adjustment for stroke severity, (3) presence of $\mathrm{HT}$ on imaging appeared to delay the resumption of OAC, and (4) the presence of $\mathrm{HT}$ in NOAC treated patients was not independently associated to an unfavourable outcome at 3 months.

Ischaemic stroke occurred under NOAC therapy in 1 to $2 \%$ of NOAC treated patients with AF per year in large randomised controlled trials. ${ }^{16-19}$ The incidence of strokes in patients taking NOACs is expected to increase in the future due to an increase of the prevalence of atrial fibrillation, ${ }^{20}$ higher utilisation of
NOAC in the population, ${ }^{21}$ and a potential extension of indications..$^{22} \mathrm{~A}$ major advantage of NOAC compared to VKA treatment is a significant reduction of intracranial haemorrhage $(\mathrm{ICH}) .^{23}$ Nevertheless, NOAC-related ICH has a similar poor prognosis as VKA-related $\mathrm{ICH}^{24}$ Three preclinical studies in mice undergoing transient middle cerebral occlusion that were anticoagulated with either warfarin or dabigatran supported the assumption that NOAC are also associated with a lower risk of HT in ischaemic stroke compared to VKA. ${ }^{25-27}$ Moreover, Bohmann and colleagues suggested that early continuation of dabigatran might be safe in experimental ischaemic stroke. ${ }^{26}$ In contrast to these experimental data, current expert guidance regarding the resumption of anticoagulation recommends starting oral anticoagulation in TIA and stroke patients with short or longer delay depending on the severity of the stroke. ${ }^{28}$

According to the present analysis, $14.0 \%(95 \% \mathrm{Cl}, 8.9$ to 21.1$)$ 
Table 3. Factors associated with early haemorrhagic transformation (univariate analysis) (follow-up imaging cohort)

\begin{tabular}{|c|c|c|}
\hline & $\mathrm{OR}(95 \% \mathrm{Cl})$ & $P$ value \\
\hline Age (year) & $0.98(0.92-1.05)$ & 0.57 \\
\hline Female sex & $0.60(0.22-1.67)$ & 0.33 \\
\hline Concomitant platelet inhibition & $2.02(0.50-8.19)$ & 0.33 \\
\hline \multicolumn{3}{|l|}{ Medical history } \\
\hline Ischaemic stroke/TIA & $0.70(0.25-1.93)$ & 0.49 \\
\hline Hypertension & $0.50(0.16-1.59)$ & 0.24 \\
\hline Hyperlipidemia & $0.63(0.21-1.90)$ & 0.41 \\
\hline Diabetes mellitus & $0.96(0.33-2.76)$ & 0.94 \\
\hline Heart failure & $1.48(0.51-4.32)$ & 0.47 \\
\hline Peripheral vascular disease & $0.76(0.09-6.45)$ & 0.80 \\
\hline Renal function: GFR $<60 \mathrm{~mL} / \mathrm{min}$ & $0.96(0.35-2.64)$ & 0.94 \\
\hline $\mathrm{CHA}_{2} \mathrm{DS}_{2}$ VASc score ${ }^{*}$ & $0.89(0.68-1.17)$ & 0.41 \\
\hline HAS-BLED score ${ }^{\dagger}$ & $0.68(0.39-1.19)$ & 0.17 \\
\hline \multicolumn{3}{|l|}{ Modified Rankin scale score ${ }^{+}$} \\
\hline Pre stroke & $1.25(0.84-1,86)$ & 0.27 \\
\hline At admission & $1.95(1.29-2.94)$ & 0.002 \\
\hline NIHSS at admission ${ }^{\S}$ & $1.09(1.03-1.16)$ & 0.006 \\
\hline ASPECTS at admission & $0.61(0.46-0.80)$ & $<0.001$ \\
\hline Infarct size & & 0.001 \\
\hline Small & Ref. & \\
\hline Medium & $15.20(2.80-82.66)$ & \\
\hline Large anterior or posterior & $19.00(3.85-93.74)$ & \\
\hline Time since last intake NOAC & $1.01(0.95-1.08)$ & 0.68 \\
\hline Time to first scan since onset" & $1.02(0.99-1.05)$ & 0.24 \\
\hline
\end{tabular}

TIA, transient ischaemic attack; GFR, glomerular filtration rate (electronic GFR as reported by the centres); NIHSS, National Institutes of Health Stroke Scale; ASPECTS, Alberta Stroke Program Early Computed Tomography Score; NOAC, non-vitamin $\mathrm{K}$ antagonist oral anticoagulant.

${ }^{*} \mathrm{CHA}_{2} \mathrm{DS}_{2}$ VASc score, range 0-9, from low to high risk of ischaemic stroke in atrial fibrillation; ${ }^{+} H A S-B L E D$ score, range $0-9$, from low to high risk of Haemorrhage under oral anticoagulation; ${ }^{*}$ Modified Rankin scale score, from 0 (no symptoms) to 6 (death); ${ }^{5} \mathrm{NIHSS}$, stroke related neurological deficits, from 0 (no symptoms) to 42; "Excluding cases with unknown onset.

of the NOAC anticoagulated patients with initial- and follow-up imaging developed $\mathrm{HT}$, which is slightly higher than reported for VKA treated patients ( $10 \%$ in patients with INR $<2$, but no data on patients with INR $>2$ available), ${ }^{5}$ and for patients without antithrombotic or thrombolysis treatment (8.5\%, 95\% Cl 7\% to 10\%). In contrast, substantially higher rates of HT have been reported in the placebo arm of intravenous thrombolysis trials (21.6 to $29.6 \%)^{29}$

Although there is an ongoing debate about the relevance of both initial clinically symptomatic and asymptomatic forms of $\mathrm{HT}$ in terms of functional outcome, ${ }^{30-33}$ the present study highlights a more direct clinical consequence: Delayed resumption of OAC after occurrence of HT. Primary or secondary prevention of ischaemic stroke in (non-valvular) atrial fibrillation was the indication of oral anticoagulation in the majority of our patients. These patients are at a high risk of recurrent stroke, indicated by a median $\mathrm{CHA}_{2} \mathrm{DS}_{2}$ VASc score of 5 at admission (excluding the
Table 4. Factors associated with unfavourable outcome (mRS 3-6) at 3-months follow up (multivariate analysis) (follow-up imaging cohort)

\begin{tabular}{lll}
\hline & \multicolumn{1}{c}{ OR $(95 \% \mathrm{Cl})$} & $P$ value \\
\hline Age (year) & $1.07(0.97-1.13)$ & 0.20 \\
Gender: female & $1.46(0.52-4.14)$ & 0.47 \\
Medical history: Hypertension & $9.81(1.92-50.04)$ & 0.006 \\
NIHSS admission (0-3 vs. $\geq 4)^{*}$ & $3.33(1.18-9.39)$ & 0.023 \\
Modified Rankin scale score pre stroke ${ }^{\dagger}$ & $1.97(1.23-3.17)$ & 0.005 \\
Infarct size & \multicolumn{1}{c}{ Ref. } & \\
$\quad$ Small & $2.40(0.61-9.35)$ & \\
$\quad$ Medium & $6.46(2.02-20.73)$ & \\
$\quad$ Large anterior or posterior & $3.26(0.66-16.04)$ & 0.14 \\
Haemorrhagic transformation & & \\
\hline
\end{tabular}

*NIHSS grouped into (0-3 vs. $\geq 4$ ) based on Baird et al., 2001. ${ }^{39}$; ${ }^{+}$Modified Rankin scale score, pre stroke from 0 (no symptoms) to 5 (severe disability).

index event). Once a patient has suffered from $\mathrm{HT}$, resumption of OAC is usually postponed until HT is resolved or at least considered radiologically stable. In our study, OAC was indeed resumed significantly later in patients in whom early-HT occurred. However, larger infarct-sizes in the HT group might have contributed to this finding.

Interestingly, the median admission NIHSS of 4 in our population was relatively low compared to cardioembolic stroke. ${ }^{34} \mathrm{~A}$ potential explanation might be that orally anticoagulated patients have less severe stroke compared to non-anticoagulated patients with $\mathrm{AF}$ in observational studies. ${ }^{35,36}$ Alternatively, stroke may have been caused by another etiology of cardioembolism.

Larger scale prospective studies are needed to determine whether early restart or continued anticoagulation in non-disabling small ischaemic stroke with less severe blood-brain barrier damage is safe and beneficial. The proportion of $9 \%$ of patients who developed HT despite small-size infarction in our cohort however still warrants caution. A recent single-centre open-label non-randomised study found no increased risk of $\mathrm{HT}$ after initiation of dabigatran therapy within 24 hours of TIA or minor stroke (NIHSS $\leq 3) .{ }^{37}$ Another single-centre open-label study reports a progression of asymptomatic $\mathrm{HT}$ after administration of rivaroxaban on a median of 3 days after TIA and mild-to-moderate stroke (NIHSS $\leq 9$ ) comparing baseline to follow-up MRI at day $7 .^{38}$

Our study has some limitations. Recruitment was imbalanced among centres with six centres recruiting $57 \%$ of patients. However, a sensitivity analysis revealed no difference in terms of age, sex, and stroke severity in patients from top-recruiting versus other centres (Supplementary Table 4). As the RASUNOA pilot study did not have a simultaneously recruited control group, only indirect comparisons with other anticoagulation or no anticoagulation are possible. We do not have data regarding intercurrent antiplatelet therapy, which might have influenced the risk of HT.

Although the present study comprises the largest prospectively 
collected observational cohort of IS patients under NOAC treatment to our knowledge, its sample size is still limited and larger scale prospective studies are needed in order to confirm the results. Nevertheless, due to its observational character with only few exclusion criteria, we expect generalisability of the findings. Finally, neither the study design nor the sample-size allowed detection of potential differences in rate and severity of $\mathrm{HT}$ related to specific NOAC agents or specific doses.

\section{Conclusions}

In conclusion, we found a similar risk of $\mathrm{HT}$ after ischemic stroke in patients on NOAC as previously reported for VKA-anticoagulated, and non-anticoagulated patients. However, replication of the results in future prospective studies using matched control groups is therefore necessary, and current indirect comparisons should be interpreted with caution. While clinicians should carefully avoid continuation of NOAC therapy in patients with large infarcts, more evidence is needed to avoid potentially unnecessary delays of restarting anticoagulation in patients with HT of small or moderate ischaemic strokes, who are at risk of recurrent thromboembolism.

\section{Acknowledgments}

We thank all principal investigators of the RASUNOA study and participating hospitals who enrolled at least one ischaemic stroke patient (A-Z). A Binder (Kiel), M Dichgans (München), R Dziewas (Münster), K Gröschel (Mainz), M Eicke (Idar-Oberstein), M Ertl (Regensburg), MG Hennerici (Mannheim), C Hobohm (Leipzig), T Höhle (Herne), S Jander (Düsseldorf), E Jüttler (Ulm), A Khaw (Greifswald), C Kleinschnitz (Würzburg), A Kraft (Halle), M Köhrmann (Erlangen), F Meisel (Karlsruhe), T Neumann-Haefelin (Fulda), C Opherk (Heilbronn), F Palm (Ludwigshafen), S Poli (Tübingen), J Röther (Hamburg), E Schmid (Stuttgart), G Seidel (Hamburg), H Sodan (Bad Neustadt), C Tanislav (Gießen), G Thomalla (Hamburg), R Veltkamp (Heidelberg), K Wartenberg (Halle-Wittenberg), C Weimar (Essen).

JCP received travel and congress participation support from Pfizer, and personal fees from Boehringer Ingelheim, outside the submitted work. TR received consulting honoraria, speakers' honoraria and travel support from BMS Pfizer, Boehringer Ingelheim, Bayer HealthCare and Daichii Sankyo, outside the submitted work. PK received consulting honoraria, speakers' honoraria, travel support or research support from Boehringer Ingelheim, Bayer HealthCare, BMS Pfizer and Daiichi Sankyo. SP received honoraria from C.R. Bard, Bayer Healthcare, BeneChill, BMSPfizer, Boehringer-Ingelheim, Daiichi Sankyo, EMCOOLS, and
ZOLL; institutional research support from BeneChill, BMS-Pfizer, Covidien, EMCOOLS, Helena Laboratories, HVM Medical, Raumedic, and ZOLL; congress/meeting traveling and accommodation costs: C.R. Bard, Bayer Healthcare, BeneChill, Boehringer-Ingelheim, EMCOOLS, and ZOLL. RD has received speaker fees and consultation honoraria from Bayer, Boehringer Ingelheim, BMS, Pfizer and Daiichi Sankyo. FP received consulting honoraria, speakers' honoraria or travel support from Boehringer Ingelheim, BMS, Pfizer and Daiichy-Sankyo. SJ received honoraria for speaking and consultancy from Boehringer Ingelheim, Bayer Healthcare, BMS, Pfizer, and Daiichi Sankyo.PUH reports grants from BMBF, EU, Charité, Berlin Chamber of Physicians, German Parkinson Society, University Hospital Würzburg, Robert-Koch-Institute, Charité-Universitätsmedizin Berlin (within MonDAFIS; MonDAFIS is supported by an unrestricted research grant to the Charité from Bayer), University Göttingen (within FIND-AFrandomised, supported by an unrestricted research grant to the University Göttingen from Boehringer-Ingelheim), and University Hospital Heidelberg (within RASUNOA-prime; supported by an unrestricted research grant to the University Hospital Heidelberg from Bayer, BMS, Boehringer-Ingelheim, Daiichi Sankyo), outside the submitted work. RV has received speaker fees, consulting honoraria and research support from Bayer, Boehringer Ingelheim, BMS, Pfizer, Daiichi Sankyo, CSL Behring. The other authors report no competing interests.

\section{References}

1. Lindley RI, Wardlaw JM, Sandercock PA, Rimdusid P, Lewis SC, Signorini DF, et al. Frequency and risk factors for spontaneous hemorrhagic transformation of cerebral infarction. J Stroke Cerebrovasc Dis 2004;13:235-246.

2. Paciaroni $M$, Agnelli $G$, Corea $F$, Ageno W, Alberti $A$, Lanari $A$, et al. Early hemorrhagic transformation of brain infarction: rate, predictive factors, and influence on clinical outcome: results of a prospective multicenter study. Stroke 2008;39:22492256.

3. Hacke W, Kaste M, Bluhmki E, Brozman M, Davalos A, Guidetti $D$, et al. Thrombolysis with alteplase 3 to 4.5 hours after acute ischemic stroke. N Engl J Med 2008;359:1317-1329.

4. Alvarez-Sabin J, Maisterra O, Santamarina E, Kase CS. Factors influencing haemorrhagic transformation in ischaemic stroke. Lancet Neurol 2013;12:689-705.

5. O'Donnell M, Oczkowski W, Fang J, Kearon C, Silva J, Bradley C, et al. Preadmission antithrombotic treatment and stroke severity in patients with atrial fibrillation and acute ischaemic stroke: an observational study. Lancet Neurol 2006;5:749-754.

6. Easton JD, Saver JL, Albers GW, Alberts MJ, Chaturvedi S, Feld- 
mann $E_{1}$ et al. Definition and evaluation of transient ischemic attack. Stroke 2009;40:2276-2293.

7. Lip GY, Lane DA, Buller H, Apostolakis S. Development of a novel composite stroke and bleeding risk score in patients with atrial fibrillation: the AMADEUS Study. Chest 2013;144:18391847.

8. Lip GY, Frison L, Halperin JL, Lane DA. Comparative validation of a novel risk score for predicting bleeding risk in anticoagulated patients with atrial fibrillation: the HAS-BLED (Hypertension, Abnormal Renal/Liver Function, Stroke, Bleeding History or Predisposition, Labile INR, Elderly, Drugs/Alcohol Concomitantly) score. J Am Coll Cardiol 2011;57:173-180.

9. Purrucker JC, Wolf M, Haas K, Rizos T, Khan S, Dziewas R, et al. Safety of Endovascular Thrombectomy in Patients Receiving Non-Vitamin K Antagonist Oral Anticoagulants. Stroke 2016; 47:1127-1130.

10. Haacke EM, Mittal S, Wu Z, Neelavalli J, Cheng YC. Susceptibility-weighted imaging: technical aspects and clinical applications, part 1. Am J Neuroradiol 2009;30:19-30.

11. Hacke $W$, Kaste $M$, Fieschi $C$, Toni $D$, Lesaffre $E_{1}$ von Kummer $R_{1}$ et al. Intravenous thrombolysis with recombinant tissue plasminogen activator for acute hemispheric stroke. The European Cooperative Acute Stroke Study (ECASS). JAMA 1995;274:10171025.

12. Hacke $W$, Kaste $M$, Fieschi $C$, von Kummer $R$, Davalos $A$, Meier $D$, et al. Randomised double-blind placebo-controlled trial of thrombolytic therapy with intravenous alteplase in acute ischaemic stroke (ECASS II). Second European-Australasian Acute Stroke Study Investigators. Lancet 1998;352:1245-1251.

13. Barber PA, Demchuk AM, Zhang J, Buchan AM. Validity and reliability of a quantitative computed tomography score in predicting outcome of hyperacute stroke before thrombolytic therapy. ASPECTS Study Group. Alberta Stroke Programme Early CT Score. Lancet 2000;355:1670-1674.

14. Puetz V, Sylaja PN, Coutts SB, Hill MD, Dzialowski I, Mueller $P_{1}$ et al. Extent of hypoattenuation on CT angiography source images predicts functional outcome in patients with basilar artery occlusion. Stroke 2008;39:2485-2490.

15. Agresti A, Coull BA. Approximate is better than "exact" for interval estimation of binomial proportions. The American Statistician 1998;52:119-126.

16. Connolly SJ, Ezekowitz MD, Yusuf S, Eikelboom J, Oldgren J, Parekh $A$, et al. Dabigatran versus warfarin in patients with atrial fibrillation. N Engl J Med 2009;361:1139-1151.

17. Patel MR, Mahaffey KW, Garg J, Pan G, Singer DE, Hacke W, et al. Rivaroxaban versus warfarin in nonvalvular atrial fibrillation. N Engl J Med 2011;365:883-891.

18. Granger CB, Alexander JH, McMurray JJ, Lopes RD, Hylek EM,
Hanna $M$, et al. Apixaban versus warfarin in patients with atrial fibrillation. N Engl J Med 2011;365:981-992.

19. Giugliano RP, Ruff CT, Braunwald E, Murphy SA, Wiviott SD, Halperin $J L$, et al. Edoxaban versus warfarin in patients with atrial fibrillation. N Engl J Med 2013;369:2093-2104.

20. Kirchhof $P$, Breithardt G, Bax J, Benninger G, Blomstrom-Lundqvist $C$, Boriani $G$, et al. A roadmap to improve the quality of atrial fibrillation management: proceedings from the fifth Atrial Fibrillation Network/European Heart Rhythm Association consensus conference. Europace 2016;18:37-50.

21. Huisman MV, Rothman KJ, Paquette M, Teutsch C, Diener HC, Dubner SJ, et al. Antithrombotic Treatment Patterns in Patients with Newly Diagnosed Nonvalvular Atrial Fibrillation: The GLORIA-AF Registry, Phase II. Am J Med 2015;128:1306-1313.

22. Hart RG, Diener HC, Coutts SB, Easton JD, Granger CB, O'Donnell $M J$, et al. Embolic strokes of undetermined source: the case for a new clinical construct. Lancet Neuro/ 2014;13:429-438.

23. Ruff CT, Giugliano RP, Braunwald E, Hoffman EB, Deenadayalu $\mathrm{N}$, Ezekowitz MD, et al. Comparison of the efficacy and safety of new oral anticoagulants with warfarin in patients with atrial fibrillation: a meta-analysis of randomised trials. Lancet 2014;383:955-962.

24. Purrucker JC, Haas K, Rizos T, Khan S, Wolf M, Hennerici MG, et al. Early Clinical and Radiological Course, Management, and Outcome of Intracerebral Hemorrhage Related to New Oral Anticoagulants. JAMA Neurol 2016;73:169-177.

25. Pfeilschifter W, Spitzer D, Czech-Zechmeister B, Steinmetz $H$, Foerch $C$. Increased risk of hemorrhagic transformation in ischemic stroke occurring during warfarin anticoagulation: an experimental study in mice. Stroke 2011;42:1116-1121.

26. Bohmann F, Mirceska A, Pfeilschifter J, Lindhoff-Last E, Stein-

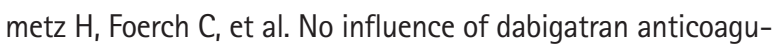
lation on hemorrhagic transformation in an experimental model of ischemic stroke. PLoS ONE 2012;7:e40804.

27. Gliem M, Hermsen D, van Rooijen N, Hartung HP, Jander S. Secondary intracerebral hemorrhage due to early initiation of oral anticoagulation after ischemic stroke: an experimental study in mice. Stroke 2012;43:3352-3357.

28. Heidbuchel $H$, Verhamme $P$, Alings $M$, Antz $M$, Hacke W, Oldgren J, et al. European Heart Rhythm Association Practical Guide on the use of new oral anticoagulants in patients with non-valvular atrial fibrillation. Europace 2013;15:625-651.

29. Lees KR, Bluhmki E, von Kummer R, Brott TG, Toni D, Grotta JC, et al. Time to treatment with intravenous alteplase and outcome in stroke: an updated pooled analysis of ECASS, ATLANTIS, NINDS, and EPITHET trials. Lancet 2010;375:1695-1703.

30. Park JH, Ko Y, Kim WJ, Jang MS, Yang MH, Han MK, et al. Is asymptomatic hemorrhagic transformation really innocuous? 
Neurology 2012;78:421-426.

31. Lei $C$, Wu B, Liu M, Chen Y. Asymptomatic hemorrhagic transformation after acute ischemic stroke: is it clinically innocuous? J Stroke Cerebrovasc Dis 2014;23:2767-2772.

32. Dzialowski I, Pexman JH, Barber PA, Demchuk AM, Buchan AM, Hill MD, et al. Asymptomatic hemorrhage after thrombolysis may not be benign: prognosis by hemorrhage type in the Canadian alteplase for stroke effectiveness study registry. Stroke 2007;38:75-79.

33. Jickling GC, Liu D, Stamova B, Ander BP, Zhan X, Lu A, et al. Hemorrhagic transformation after ischemic stroke in animals and humans. J Cereb Blood Flow Metab 2014;34:185-199.

34. Kimura K, Minematsu K, Yamaguchi T, Japan Multicenter Stroke Investigators $C$. Atrial fibrillation as a predictive factor for severe stroke and early death in 15,831 patients with acute ischaemic stroke. J Neurol Neurosurg Psychiatry 2005;76:679683.

35. Hylek EM, Go AS, Chang Y, Jensvold NG, Henault LE, Selby JV, et al. Effect of intensity of oral anticoagulation on stroke severity and mortality in atrial fibrillation. N Engl J Med 2003; 349:1019-1026.

36. Audebert HJ, Schenk B, Schenkel J, Heuschmann PU. Impact of prestroke oral anticoagulation on severity and outcome of ischemic and hemorrhagic stroke in patients with atrial fibrillation. Cerebrovasc Dis 2010;29:476-483.

37. Kate M, Gioia L, Buck B, Sivakumar L, Jeerakathil T, Shuaib A, et al. Dabigatran Therapy in Acute Ischemic Stroke Patients Without Atrial Fibrillation. Stroke 2015;46:2685-2687.

38. Gioia LC, Kate M, Sivakumar L, Hussain D, Kalashyan H, Buck $B$, et al. Early Rivaroxaban Use After Cardioembolic Stroke May Not Result in Hemorrhagic Transformation: A Prospective Magnetic Resonance Imaging Study. Stroke 2016;47:19171919.

39. Baird $A E$, Dambrosia J, Janket $S$, Eichbaum $Q$, Chaves $C$, Silver $B$, et al. A three-item scale for the early prediction of stroke recovery. Lancet 2001;357:2095-2099. 
Supplementary Table 1. Clinical characteristics and outcome of patients with and without recanalisation therapy (only patients with available follow-up imaging)

\begin{tabular}{|c|c|c|c|}
\hline & $\begin{array}{c}\text { Patients without recanalization } \\
\text { therapy }(\mathrm{N}=129)\end{array}$ & $\begin{array}{c}\text { Patients with recanalization } \\
\text { therapy }(\mathrm{N}=32)\end{array}$ & $P$ value \\
\hline Age (year) & $77.0( \pm 7.9)$ & $75.7( \pm 11.7)$ & 0.54 \\
\hline Women & $64(49.6)$ & $17(53.1)$ & 0.84 \\
\hline NOAC & & & 0.78 \\
\hline Apixaban & $20(15.5)$ & $5(15.6)$ & \\
\hline Dabigatran & $36(27.9)$ & $7(21.9)$ & \\
\hline Rivaroxaban & $73(56.6)$ & $20(62.5)$ & \\
\hline Concomitant platelet inhibition & $13(10.1)$ & $3(9.4)$ & $>0.99$ \\
\hline $\mathrm{CHA}_{2} \mathrm{DS}_{2}$ VASc score & $5(3-6)$ & $4(3-5)$ & 0.02 \\
\hline HAS-BLED score & $4(3-4)$ & $3(3-3)$ & $<0.001$ \\
\hline \multicolumn{4}{|l|}{ Medical history } \\
\hline Ischemic stroke/TIA & $60(46.5)$ & $6(18.8)$ & 0.005 \\
\hline Intracranial haemorrhage & $3(2.3)$ & $0(0)$ & $>0.99$ \\
\hline Atrial fibrillation & $119(92.2)$ & $26(81.3)$ & 0.09 \\
\hline Hypertension & $106(82.2)$ & $26(81.3)$ & $>0.99$ \\
\hline Hyperlipidemia & $47(36.4)$ & 7 (21.9) & 0.15 \\
\hline Diabetes mellitus & $44(34.1)$ & 4 (12.5) & 0.02 \\
\hline Heart failure & $34(26.4)$ & $10(31.3)$ & 0.66 \\
\hline Peripheral vascular disease & $9(7.0)$ & $1(3.1)$ & 0.69 \\
\hline \multicolumn{4}{|l|}{ Renal function } \\
\hline $\mathrm{GFR}<60 \mathrm{~mL} / \mathrm{min}$ & $53(41.1)$ & $10(31.3)$ & 0.30 \\
\hline Creatinin level (mg/dL) & $1.01(0.87-1.30)$ & $0.9(0.79-1.08)$ & 0.02 \\
\hline NIHSS at admission & $4(2-7)$ & $19(14-22)$ & $<0.001$ \\
\hline \multicolumn{4}{|l|}{ Modified Rankin scale score } \\
\hline Pre stroke & $1(0-2)$ & $1(0-2)$ & 0.24 \\
\hline At admission & $3(1-4)$ & $5(5-5)$ & $<0.001$ \\
\hline At discharge & $2(1-4)$ & $4(3-5)$ & $<0.001$ \\
\hline At 3-months* & $2(1-4)$ & $4(3-6)$ & $<0.001$ \\
\hline mRS 0-2 at 3 months & 66 (51.2) & $5(15.6)$ & $<0.001$ \\
\hline In-hospital mortality & $4(3.1)$ & $3(9.4)$ & 0.14 \\
\hline Time since last intake NOAC (hour) & $9(4-18)$ & $10(5-24)$ & 0.45 \\
\hline \multicolumn{4}{|l|}{ Admission imaging } \\
\hline ASPECTS & $10(9-10)$ & $8(7-10)$ & $<0.001$ \\
\hline Haemorrhagic transformation & & & 0.54 \\
\hline $\mathrm{HI}$ & 2 & 0 & \\
\hline $\mathrm{HI} 2$ & 3 & 0 & \\
\hline $\mathrm{PH} 1$ & 0 & 0 & \\
\hline $\mathrm{PH} 2$ & 0 & 0 & \\
\hline $\mathrm{PHr} 1 / \mathrm{PHr} 2$ & $0 / 0$ & $0 / 0$ & \\
\hline Other haemorrhage & & & - \\
\hline SAH & 0 & 0 & \\
\hline IVH & 0 & 0 & \\
\hline $\mathrm{sICH}$ & 0 & 0 & - \\
\hline \multicolumn{4}{|l|}{ Follow-up imaging until day 7} \\
\hline ASPECTS & $9(8-10)$ & $6(3-8)$ & $<0.001$ \\
\hline Infarct size & & & $<0.001$ \\
\hline Small/medium & 99 (76.7) & 10 (31.3) & \\
\hline Large anterior/posterior & $30(23.3)$ & $22(68.8)$ & \\
\hline Haemorrhagic transformation & $18(14.0)$ & $13(40.6)$ & 0.002 \\
\hline Haemorrhagic transformation & & & 0.03 \\
\hline $\mathrm{HI}$ & 10 & 5 & \\
\hline $\mathrm{HI} 2$ & 2 & 3 & \\
\hline $\mathrm{PH} 1$ & 0 & 5 & \\
\hline $\mathrm{PH} 2$ & 1 & 1 & \\
\hline $\mathrm{PHr} 1 /$ PHr2 & $0 / 0$ & $0 / 0$ & \\
\hline
\end{tabular}


Supplementary Table 1. Continued from the previous page

\begin{tabular}{lccc}
\hline & $\begin{array}{c}\text { Patients without recanalization } \\
\text { therapy }(\mathrm{N}=129)\end{array}$ & $\begin{array}{c}\text { Patients with recanalization } \\
\text { therapy }(\mathrm{N}=32)\end{array}$ & $P$ value \\
\hline Other haemorrhage & 0 & 2 & 0.04 \\
SAH & 0 & 1 & - \\
IVH & 1 & 1 & - \\
SICH & & & \\
\hline
\end{tabular}

Data are mean $( \pm S D)$, median $(I Q R)$ or $n(\%)$.

*3-months modified Rankin data available for $n=117 / 129$ (90.7\%, patients without recanalization therapy), and $n=31 / 32$ patients (96.9\%, patients with recanalization therapy). 
Supplementary Table 2. Baseline clinical and radiological characteristics of patients with and without follow up imaging

\begin{tabular}{|c|c|c|c|}
\hline & Patients with FU img. $(\mathrm{N}=129)$ & Patients without FU img. $(\mathrm{N}=102)$ & $P$ value \\
\hline Age (year) & $77.0( \pm 7.9)$ & $77.9( \pm 9.0)$ & 0.43 \\
\hline Women & $64(49.6)$ & $44(43.1)$ & 0.35 \\
\hline NOAC & & & 0.84 \\
\hline Apixaban & $20(15.5)$ & $16(15.7)$ & \\
\hline Dabigatran & $36(27.9)$ & $26(25.5)$ & \\
\hline Rivaroxaban & $73(56.6)$ & $60(58.8)$ & \\
\hline Concomitant platelet inhibition & $13(10.1)$ & $11(10.8)$ & $>0.99$ \\
\hline $\mathrm{CHA}_{2} \mathrm{DS}_{2}$ VASc score & $5(3-6)$ & $4.5(3-6)$ & 0.59 \\
\hline HAS-BLED score & $4(3-4)$ & $4(3-4)$ & 0.30 \\
\hline \multicolumn{4}{|l|}{ Medical history } \\
\hline Ischemic stroke/TIA & $60(46.5)$ & $47(46.1)$ & $>0.99$ \\
\hline Intracranial hemorrhage & $3(2.3)$ & $2(2.0)$ & $>0.99$ \\
\hline Atrial fibrillation & $119(92.2)$ & $90(88.2)$ & 0.37 \\
\hline Hypertension & $106(82.2)$ & $87(85.3)$ & 0.59 \\
\hline Hyperlipidemia & $47(36.4)$ & $34(33.3)$ & 0.68 \\
\hline Diabetes mellitus & $44(34.1)$ & $25(24.5)$ & 0.15 \\
\hline Heart failure & $34(26.4)$ & 19 (18.6) & 0.21 \\
\hline Peripheral vascular disease & $9(7.0)$ & $6(5.9)$ & 0.79 \\
\hline Renal function & & & 0.20 \\
\hline $\mathrm{GFR}<60 \mathrm{~mL} / \mathrm{min}$ & $53(41.1)$ & $32(31.4)$ & \\
\hline Creatinin level (mg/dL) & $1.01(0.87-1.30)$ & $1.02(0.84-1.28)$ & 0.99 \\
\hline NIHSS at admission & $4(2-7)$ & $3(2-6)$ & 0.36 \\
\hline \multicolumn{4}{|l|}{ Modified Rankin scale score } \\
\hline Pre stroke & $1(0-2)$ & $1(0-2)$ & 0.09 \\
\hline At admission & $3(1-4)$ & $3(2-4)$ & 0.87 \\
\hline At discharge & $2(1-4)$ & $2(2-3)$ & 0.57 \\
\hline At 3-months & $2(1-4)$ & $3(1-4)$ & 0.33 \\
\hline mRS 0-2 at 3 months & $66(56.4)$ & $44(48.9)$ & 0.33 \\
\hline In-hospital mortality & $4(3.1)$ & $2(2.0)$ & 0.70 \\
\hline Time since last intake NOAC (hour) & $9(4.2-18.3)$ & $8.3(4.5-13.1)$ & 0.35 \\
\hline Baseline ASPECTS & $10(9-10)$ & $10(9-10)$ & 0.55 \\
\hline Infarct size & & & 0.06 \\
\hline Small/medium & 99 (76.7) & 89 (87.3) & \\
\hline Large anterior/posterior & $30(23.3)$ & $13(12.7)$ & \\
\hline
\end{tabular}


Supplementary Table 3. Factors associated with unfavourable outcome (mRS 3-6) at 3-months follow up (univariate analysis; follow-up imaging cohort)

\begin{tabular}{|c|c|c|}
\hline & OR (95 \% Cl) & $P$ value \\
\hline Age (year) & $1.08(1.02-1.14)$ & 0.005 \\
\hline Female sex & $1.58(0.76-3.30)$ & 0.22 \\
\hline Concomitant platelet inhibition & $1.09(0.31-3.78)$ & 0.90 \\
\hline \multicolumn{3}{|l|}{ Medical history } \\
\hline Ischaemic stroke/TIA & $1.41(0.68-2.94)$ & 0.36 \\
\hline Atrial fibrillation & $0.59(0.15-2.33)$ & 0.46 \\
\hline Hypertension & $4.71(1.28-17.28)$ & 0.020 \\
\hline Hyperlipidemia & $1.29(0.60-2.76)$ & 0.51 \\
\hline Diabetes mellitus & $3.27(1.48-7.24)$ & 0.004 \\
\hline Heart failure & $2.04(0.88-4.73)$ & 0.097 \\
\hline Peripheral vascular disease & $1.68(0.43-6.62)$ & 0.46 \\
\hline Renal function: GFR $<60 \mathrm{~mL} / \mathrm{min}$ & $2.13(0.98-4.66)$ & 0.058 \\
\hline $\mathrm{CHA}_{2} \mathrm{DS}_{2}$ VASc score ${ }^{*}$ & $1.57(1.21-2.03)$ & 0.001 \\
\hline HAS-BLED score ${ }^{+}$ & $1.91(1.17-3.11)$ & 0.010 \\
\hline \multicolumn{3}{|l|}{ Modified Rankin scale score ${ }^{\dagger}$} \\
\hline Pre stroke & $2.29(1.54-3.39)$ & $<0.001$ \\
\hline At admission & $2.60(1.85-3.65)$ & $<0.001$ \\
\hline NIHSS at admission ${ }^{\S}$ & $1.29(1.15-1.45)$ & $<0.001$ \\
\hline ASPECTS at admission & $0.51(0.34-0.76)$ & 0.001 \\
\hline \multicolumn{3}{|l|}{ Infarct size } \\
\hline Small & Ref. & 0.001 \\
\hline Medium & $1.36(0.46-3.98)$ & \\
\hline Large anterior or posterior & $5.88(2.26-15.26)$ & \\
\hline Time since last intake NOAC & $1.00(0.95-1.05)$ & 0.99 \\
\hline Time to first scan since onset" & $1.00(1.00-1.00)$ & 0.66 \\
\hline Haemorrhagic transformation & 3.08 (1.07-8.88) & 0.038 \\
\hline
\end{tabular}

${ }^{*} \mathrm{CHA}_{2} \mathrm{DS}_{2} \mathrm{VASc}$ score, range 0-9, from low to high risk of ischaemic stroke in atrial fibrillation; ${ }^{+} \mathrm{HAS}-\mathrm{BLED}$ score, range $0-9$, from low to high risk of Haemorrhage under oral anticoagulation.; ${ }^{*}$ Modified Rankin scale score, from 0 (no symptoms) to 6 (death); ${ }^{5} \mathrm{NIHSS}$, stroke related neurological deficits, from 0 (no symptoms) to 42; "Excluding cases with unknown onset. 
Supplementary Table 4. Characteristics of Patients from top-recruiting centres versus standard recruiting centres

\begin{tabular}{lccc}
\hline & $\begin{array}{c}\text { Patients from } \\
\text { top-recruiting } \\
\text { centres }\end{array}$ & $\begin{array}{c}\text { Patients from } \\
\text { standard } \\
\text { recruiting } \\
\text { centres }\end{array}$ & Pvalue \\
\hline Age (year) & $76.9( \pm 8.9)$ & $77.7( \pm 8.7)$ & 0.43 \\
Women & $73(48.3)$ & $52(46.4)$ & 0.80 \\
Pre-stroke mRS & $1(0-2)$ & $1(0-2)$ & 0.20 \\
Stroke severity & $4(2-11)$ & $4(2-7)$ & 0.15 \\
$\quad$ NIHSS at admission & $3(2-5)$ & $3(1-4)$ & 0.10 \\
$\quad$ mRS at admission & $9(8-10)$ & $10(9-10)$ & 0.34 \\
$\quad$ ASPECTS at admission & & & \\
Outcome & $13(8.6)$ & $10(8.9)$ & .93 \\
$\quad$ Early HT & $61(45.9)$ & $54(51.4)$ & .39 \\
\hline mRS 0-2 at 3-months & &
\end{tabular}

Data are mean $( \pm S D)$, median (IQR) or $n(\%)$.

$\mathrm{N}=263$, main analysis group (no-RT group) plus patients treated with recanalisation therapies. 